\title{
Nonalcoholic fatty liver disease as a predictor of atrial fibrillation: a systematic review and meta-analysis
}

\author{
Yaqiong Zhou ${ }^{1}$, Chenglin Lai ${ }^{2}$, Chunrong Peng ${ }^{3}$, Mingyue Chen ${ }^{4}$, Bolin $\mathrm{Li}^{1}$, Xiaoyun Wang ${ }^{5}$ Jingjing Sun ${ }^{6}$, \\ Chaofeng Sun ${ }^{1}$ \\ ${ }^{1}$ Department of Cardiology, the First Affiliated Hospital of Xi'an Jiaotong University, Xi'an, Shaanxi Province, China \\ 2Department of Endocrinology, the First Affiliated Hospital of Xi'an Jiaotong University, Xi'an, Shaanxi Province, China \\ ${ }^{3}$ Department of the Obstetrics and Gynecology, the First Affiliated Hospital of Xi'an Jiaotong University, Xi'an, Shaanxi Province, China \\ ${ }^{4}$ Department of Emergency Medicine, the First Affiliated Hospital of Xi'an Jiaotong University, Xi'an, Shaanxi Province, China \\ ${ }^{5}$ Department of Gastroenterology, the First Affiliated Hospital of Xi'an Jiaotong University, Xi'an, Shaanxi Province, China \\ ${ }^{6}$ Intensive Care Unit, the First Affiliated Hospital of Xi'an Jiaotong University, Xi'an, Shaanxi Province, China
}

Adv Interv Cardiol 2017; 13, 3 (49): 250-257 DOI: https://doi.org/10.5114/aic.2017.70198

\begin{abstract}
Introduction: Numerous epidemiologic studies have investigated the link between nonalcoholic fatty liver disease (NAFLD) and long-term atrial fibrillation (AF) risk, but the results are surprisingly conflicting.

Aim: Therefore, we systematically reviewed all published studies assessing the risk of AF in patients with NAFLD and conducted a meta-analysis.

Material and methods: We performed a literature search using PubMed, EMBASE and Cochrane Library databases in February 2017 with no restrictions. Two cohort studies and two cross-sectional studies were identified, involving a total of 5150 subjects (NAFLD: 1655; controls: 3495 ) in this meta-analysis. Data from selected studies were extracted and a meta-analysis was performed using a random effects model.

Results: Nonalcoholic fatty liver disease patients had a significantly higher risk of AF compared to controls (relative risk (RR): 2.61; $95 \%$ confidence interval (CI): 1.34-5.06, $\left.p=0.00 ; l^{2}=52.5 \%, p=0.097\right)$. In a further analysis stratified by presence of type 2 diabetes, the increased risk was present predominantly in patients with type 2 diabetes ( $R R=5.10 ; 95 \% \mathrm{Cl}: 2.43-10.7, p<0.001$; $R^{2}=0, p=0.958$ ). However, subjects without type 2 diabetes were at slightly increased risk of AF but the relative risk did not reach statistical significance ( $\left.\mathrm{RR}=1.68 ; 95 \% \mathrm{Cl}: 0.99-2.82, p=0.05 ; p^{2}=0, p=0.461\right)$.

Conclusions: Our meta-analysis suggested that ultrasound-diagnosed NAFLD patients have a significantly higher risk for AF after adjustment for numerous important clinical risk factors for AF. These results need to be confirmed in large prospective studies.
\end{abstract}

Key words: nonalcoholic fatty liver disease, nonalcoholic steatohepatitis, nonalcoholic cirrhosis, atrial arrhythmia, atrial fibrillation, atrial flutter.

\section{Introduction}

The last three decades have witnessed the prevalence of nonalcoholic fatty liver disease (NAFLD) rise with the growing epidemic of overweight and diabetes individuals in all countries, various age groups, ethnicities and races. According to the latest figures, NAFLD affects up to $35 \%$ of the general population and its prevalence increases to approximately $60 \%$ to $70 \%$ of persons with type 2 diabetes, who are also at increased risk for the development of more severe forms of NAFLD, such as nonalcoholic ste- atohepatitis (NASH), cirrhosis and hepatocellular carcinoma, making it a lethal disease [1-6].

Accumulating clinical and epidemiological evidence indicates that NAFLD is associated not only with liver-related morbidity and mortality but also with systematic disease, being a well-established risk factor of cardiovascular diseases, coronary heart disease (CHD), abnormalities of cardiac function and structure (e.g., heart failure, left ventricular dysfunction and hypertrophy), valvular heart disease (e.g., aortic valve sclerosis), arrhythmias and stroke [7-12]. Haddad et al. previously reported

\section{Corresponding author:}

Prof. Chaofeng Sun, Department of Cardiology, the First Affiliated Hospital of Xi'an Jiaotong University, 277 Yanta West Road, Xi'an 710061, Shaanxi Province, China, phone: +86 15102988529, e-mail: m15102988529@163.com

Received: 4.06.2017, accepted: 19.08.2017. 
a meta-analysis and revealed that NAFLD patients had a significantly higher risk for clinical cardiovascular events compared to those without $(\operatorname{RR}=1.77)[13,14]$. Nonalcoholic fatty liver disease and cardiovascular disease (CVD) share some risk factors including dyslipidemia, insulin resistance, obesity, diabetes, endothelial dysfunction, inflammation and accelerated atherosclerosis [15]. Furthermore, most of the risk factors for NAFLD patients are also independent risk factors for the development of atrial fibrillation. It has generally been assumed that NAFLD women have a higher risk of $A F$, because they have multiple common risk factors and comorbidities described above.

The past few years have seen rapidly growing interest in testing this hypothesis. Many epidemiologic studies have investigated the link between NAFLD and long-term AF risk, but the results are surprisingly conflicting. Therefore, we systematically reviewed all published studies assessing the risk of AF in patients with NAFLD and conducted a meta-analysis.

\section{Material and methods}

\section{Research objectives}

The study endpoint was atrial fibrillation (AF). The diagnosis of AF was made on the basis of the surface standard 12-lead electrocardiogram (ECG) and medical history. A 24-h Holter monitor examination was not performed.

\section{Search strategy}

The meta-analysis was performed according to the PRISMA guidelines. We searched the PubMed, EMBASE and Cochrane Library databases from inception to January 2017 with no restrictions using the following terms in combination, as MeSH or Emtree terms and text words: "nonalcoholic fatty liver disease", "nonalcoholic steatohepatitis", "nonalcoholic cirrhosis", "atrial arrhythmia", "atrial fibrillation", "atrial flutter". We searched articles published in the English language and the references of the relevant studies were also searched.

\section{Inclusion criteria}

Cohort studies and cross-sectional studies published as original articles (case reports and reviews were excluded) were considered for inclusion if they met the following criteria: (1) They presented estimates of relative risk (RR) and 95\% confidence intervals (Cls) or reported data necessary to calculate these (i.e., variance, standard error or $p$-value of the significance of the estimate). (2) Identified studies had to be independent. In case of multiple reports on the same population or subgroup of population, the most recent or comprehensive information was used. (3) Included studies were published in the English language. Studies without a control group were excluded.

\section{Data abstraction and quality assessment}

Two authors (Zhou YQ, Lai CL) independently reviewed all potentially eligible studies and extracted the data. Any disagreement or uncertainty was resolved by discussion and consensus. The following data were extracted from each study: first author's name, publication year, geographical location, mean age, sample size, study design, proportions of women, total number of subjects, number of events, summary findings, confounding factors that were adjusted for in the analysis, and RR or hazard ratios with $95 \% \mathrm{Cls}$. When multiple effect estimates were reported, we used the most comprehensively adjusted risk estimates. The Newcastle-Ottawa quality assessment scale (NOS) ranging from 0 to 9 points was used to assess the quality of identified studies based on the components of selection (0-4 points), comparability ( $0-2$ points), and exposure or outcome (0-3 points) and a total score of seven or more was considered as a high-quality study [16].

\section{Statistical analysis}

When multiple effect estimates were reported, we used the most comprehensively adjusted risk estimates reported in the original manuscript for the meta-analysis. When the actual RR was not available, we calculated the RR and 95\% Cls using Stata (College Station, TX) version 13.0. Summary RRs $(95 \% \mathrm{Cl})$ were calculated by pooling the study-specific estimates using a random-effects model because significance heterogeneity was anticipated among studies. Pooled RRs were expressed with $95 \% \mathrm{Cls}$. Statistical heterogeneity was assessed using the $R^{2}$ statistic, applying the following interpretation for $R^{2}:<50 \%=$ low heterogeneity; $50-75 \%=$ moderate heterogeneity; $>75 \%=$ high heterogeneity. Subgroup analyses were conducted to investigate potential sources of between-study heterogeneity. An exclusion sensitivity analysis was included when necessary. The potential for publication bias was examined by the funnel plot. We used STATA, version 13.0 (Stata Corp, College Station, TX) for all analyses. Statistical tests were 2 sided and used a significance level of $p<0.05$.

\section{Results}

\section{Study selection}

With the search strategy, 91 citations were initially retrieved. After removal of the 6 duplicates, a total of 85 references were screened by title and abstracts. After screening, 40 studies were identified as potentially eligible and the full text was retrieved for detailed evaluation. Twenty articles with no relevant outcomes were excluded and 16 articles were excluded because they were review and case report, leaving four studies for final inclusion in the meta-analysis [17-21]. The study flow diagram is shown in Figure 1. 


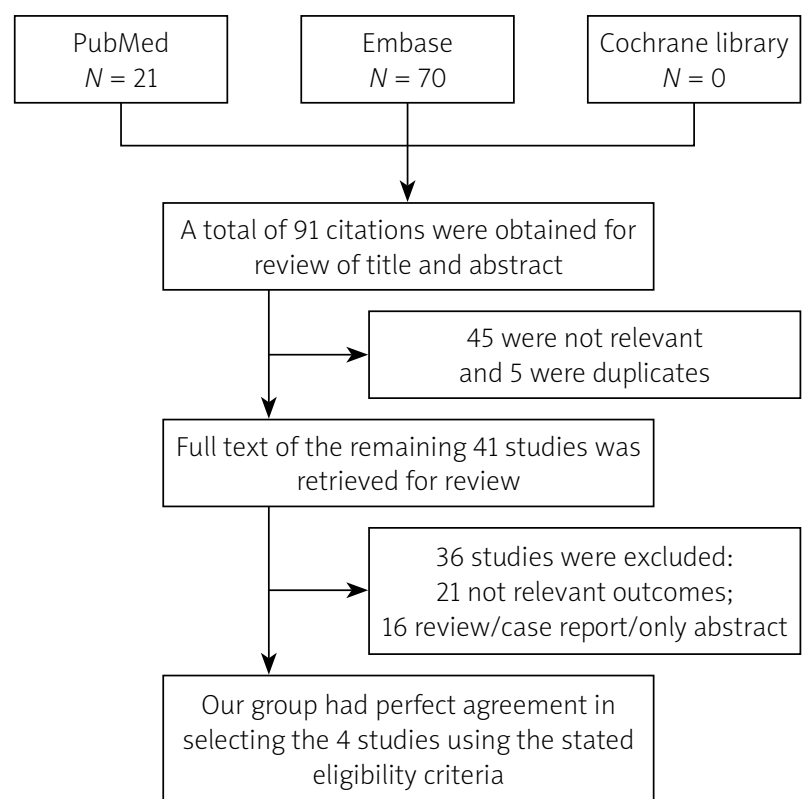

Figure 1. Study flow diagram. The flow diagram documents the flow of relevant study identification and the selection process for review and final inclusion in the meta-analysis

\section{Study characteristics}

A total of 5150 subjects (NAFLD: 1655; controls: 3495) were included in the 4 eligible studies. The sample sizes ranged from 188 to 937 cases with NAFLD and from 119 to 2153 for controls. All studies were based in Europe. Two studies recruited subjects with type 2 diabetes. There were 2 population-based cohort studies and 2 cross-sectional studies. Average follow-up duration ranged from 10 to 16.3 years. Adjusted RRs could be determined for all studies. Most risk estimates were adjusted for age, sex, body mass index (BMI), heart failure, serum creatinine, obesity, blood pressure (BP), impaired fasting glucose, dyslipidemia and so on. Study characteristics are summarized in Table I.

\section{Association of NAFLD and risk of AF}

In the NAFLD group, there were a total of $138 \mathrm{AF}$ (8.34\%), and in the non-NAFLD group, there were a total of 129 AF (3.70\%). Nonalcoholic fatty liver disease patients had a significantly higher risk of AF compared to controls $(\mathrm{RR}=2.61 ; 95 \% \mathrm{Cl}: 1.34-5.06, p=0.00)$ (Figure 2). Significant heterogeneity was present in the identified studies for the outcome of $\mathrm{AF}\left(R^{2}=52.5 \%\right.$, $p=0.097)$. In a further analysis stratified by presence of type 2 diabetes, the increased risk was present predominantly in patients with type 2 diabetes $(R R=5.10$; 95\% Cl: 2.43-10.7, $p<0.001$ ). However, subjects without type 2 diabetes were at slightly increased risk of AF but the relative risk did not reach statistical significance $(\mathrm{RR}=1.68 ; 95 \% \mathrm{Cl}: 0.99-2.82, p=0.05)$ (Figure 3). Furthermore, in the subgroup analysis according to presence of type 2 diabetes, the significant heterogeneity disappeared both in patients with and those without type 2 diabetes.

Visual inspection of the funnel plot suggested symmetry indicating no publication bias (data not shown). Publication bias was not assessed because of the presence of only four studies, which was far below the threshold of ten studies needed for assessment of publication bias. Sensitivity analysis excluding one study at a time confirmed, in direction and magnitude of statistical significance, the results from the overall analysis, which suggested that the results of this study were statistically reliable. We did not conduct a meta-regression model to explore the source of between-study heterogeneity due to the limited number of studies. Fortunately, subgroup analysis detected the source of heterogeneity and the heterogeneity was removed.

\section{Discussion}

This meta-analysis assessed the role of NAFLD as detected by ultrasonography (which is a more accurate measure in sensitivity and specificity for the detection of moderate and severe steatosis of liver fat than serum transaminase levels) in predicting development of AF [22]. The major finding of our study was that NAFLD patients have a significantly higher risk for AF and the relative risk was extremely high in patients with type 2 diabetes even after adjustment for numerous clinical risk factors for AF. Six out of 10 deaths from cardiovascular disease can be prevented by a healthy life style and dealing with preexisting risk factors. Hence it is even more important that gastroenterologists who start seeing NAFLD especially complicated with diabetes at an earlier stage initiate risk mitigation treatments than cardiologists should be aware of AF.

A systematic review and meta-analysis of seven cross-sectional studies, involving a total of 3497 subjects, suggested that NAFLD was strongly associated with increased carotid-artery intimal medial thickness and an increased prevalence of carotid atherosclerotic plaques [23]. Two previous meta-analyses from Haddad et al. showed that NAFLD was associated with increased risk of clinical cardiovascular disease, cardiovascular events and stroke, but they did not refer to the association between NAFLD and $\operatorname{AF}[13,14]$. Several studies have suggested that there were positive and independent associations between circulating levels of liver enzymes (alanine aminotransferase (ALT), aspartate aminotransferase (AST) and $\gamma$-glutamyltranspeptidase $(\gamma-\mathrm{GT}))$ and the risk of AF in adult individuals [19, 24-27]. A prior published meta-analysis from Minhas et al. [28] indicated that the risk in NAFLD was significantly increased for $A F(O R=2.47)$, although only three studies met the inclusion criteria, involving a total of 1044 subjects in their meta-analysis. Another caution is that all three identified studies reported AF with $R^{2}$ values of $60.9 \%(p=0.078)$ for pooled data, indicating signif- 


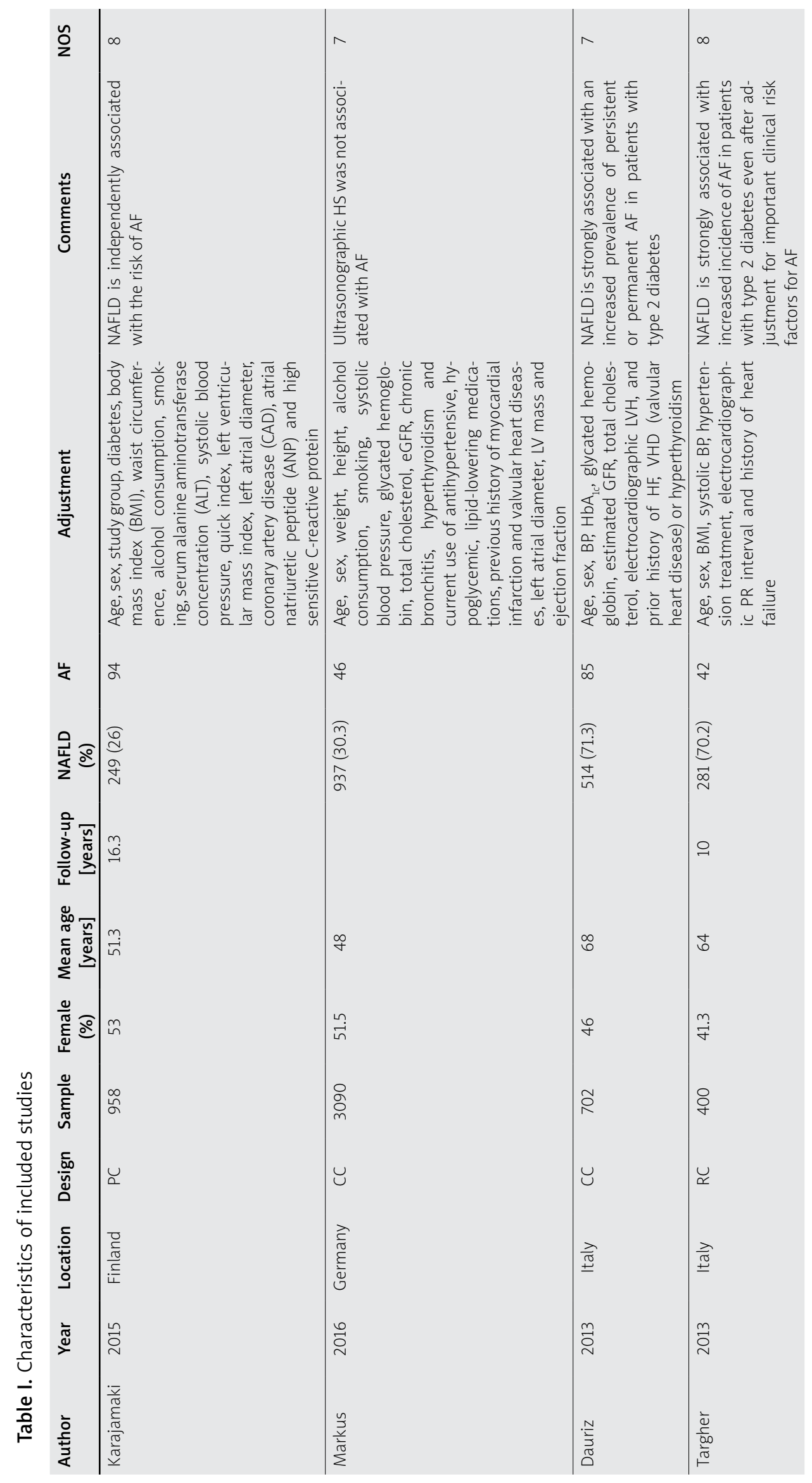




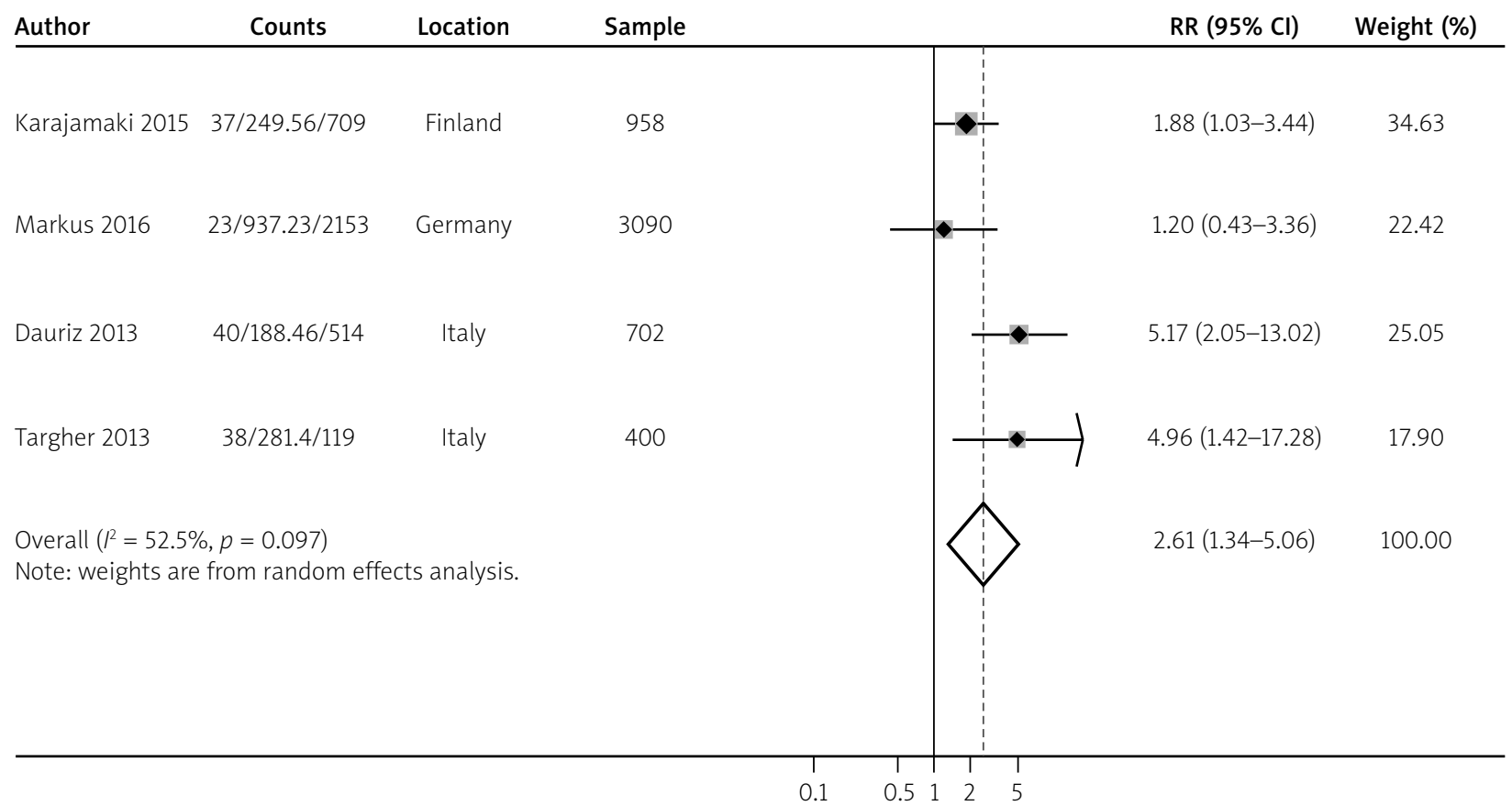

Figure 2. Forest plot showing the association of NAFLD and risk of AF. The NAFLD patients had a significantly higher risk of AF compared to controls

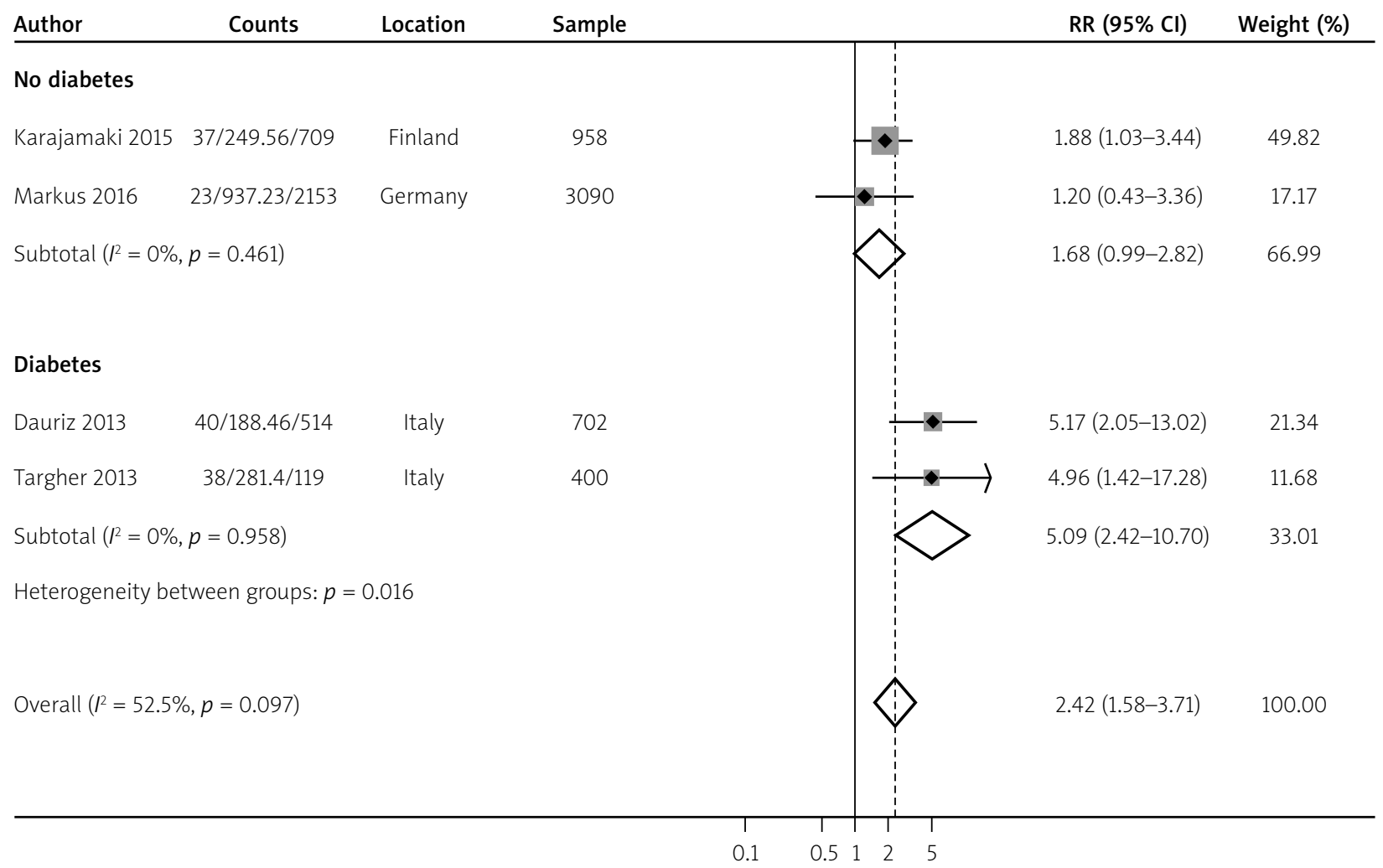

Figure 3. Forest plot showing the subgroup analysis. In a further analysis stratified by presence of type 2 diabetes, the increased risk was present predominantly in patients with type 2 diabetes. However, subjects without type 2 diabetes were at slightly increased risk of AF but the relative risk did not reach statistical significance 
icant between-study heterogeneity. However, the authors did not explore the source of heterogeneity.

The increased risk was present predominantly in the diabetes group. What are the implications of the results? As a high-prevalence health problem, diabetes mellitus has long been recognized as a risk factor for AF as well as advancing age, hypertension and congestive heart failure in the Framingham study $[29,30]$. Moreover, recent evidence points toward increased prevalence of AF in patients with diabetes. Potential reasons for this association could be some shared pathogenic factors such as insulin resistance and hypertension. It has been previously shown that traditional risk factors for AF such as dyslipidemia, hypertension, and insulin resistance are more prevalent in NAFLD patients with diabetes than those without diabetes.

The underlying pathological mechanisms responsible for the association between NAFLD and increased risk of $A F$ require further research. Whether this association is a causative one or whether these two entities just share common pathophysiologic mechanisms is unclear. Aside from shared traditional risk factors, experimental evi- dence suggests that NAFLD itself, especially nonalcoholic cirrhosis, exacerbates systemic (or hepatic) insulin resistance, causes atherogenic dyslipidemia, and releases a variety of pro-inflammatory, pro-coagulant and pro-fibrogenic mediators, e.g. C-reactive protein (CRP), interleukin-6 (IL-6), tumor necrosis factor $\alpha$ (TNF- $\alpha$ ), and other inflammatory cytokines that may play important roles in the pathophysiology of cardiac and arrhythmic complications [8, 31-34]. Furthermore, NAFLD is a marker of ectopic fat accumulation in other tissues, especially in the myocardium and pericardium, which were associated with both increased left atrial dimensions and increased prevalence of AF [35-37] (Figure 4).

The present meta-analysis has several limitations. First, our diagnosis of NAFLD was defined by ultrasonography (a method not able to detect fatty infiltration below 33\%), but was not confirmed by liver biopsy or magnetic resonance imaging $[38,39]$. This could have resulted in misclassification bias where subjects with mild NAFLD could be classified as normal, which should have resulted in underestimation rather than overestimation of the risk of AF in NAFLD. Second, the included studies

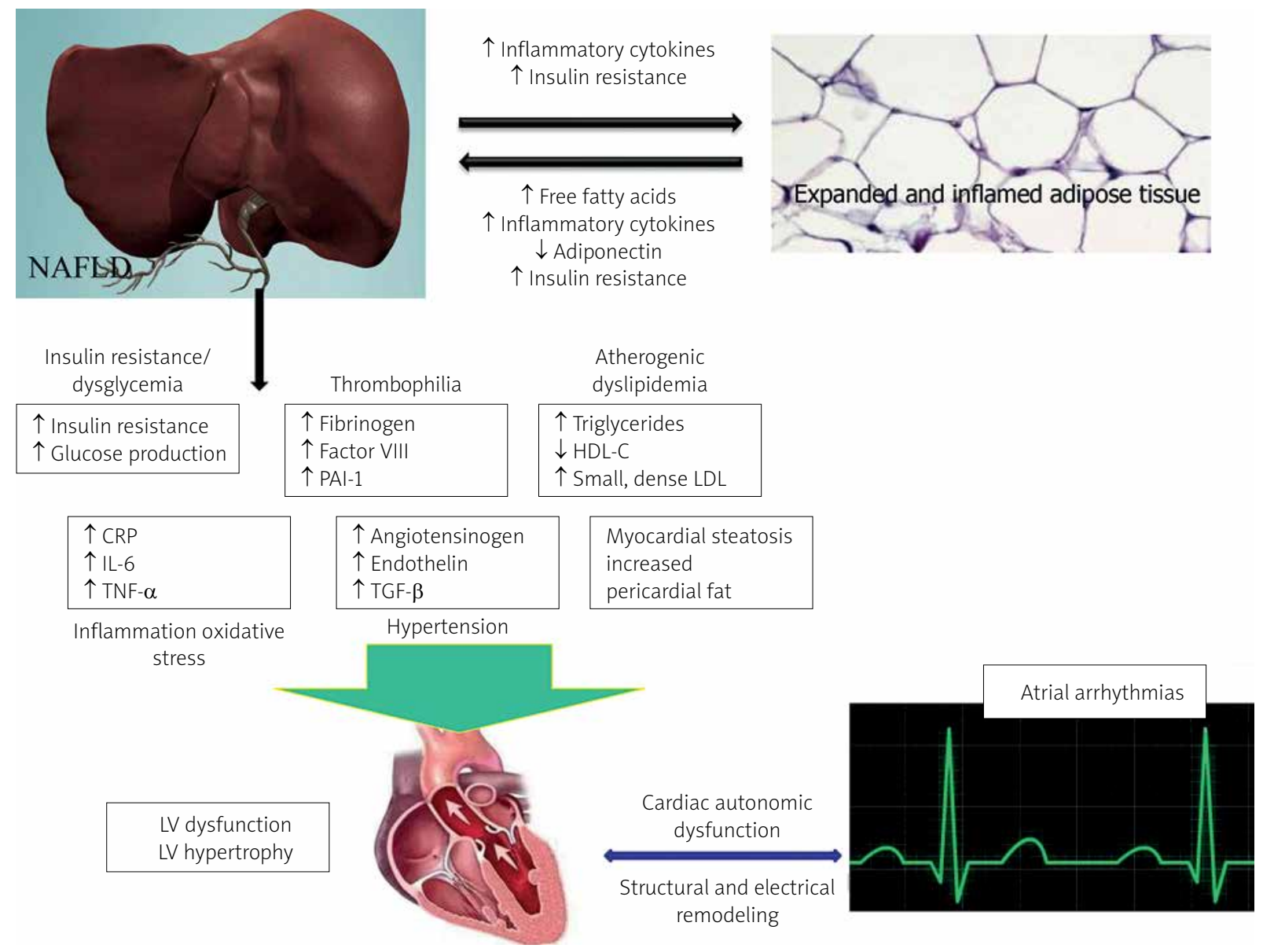

Figure 4. Possible pathological mechanisms responsible for the association between NAFLD and increased risk of $A F$

HDL-C - high-density lipoprotein cholesterol, LDL - low-density lipoprotein, CRP - C-reactive protein, IL - interleukin, TNF - tumor necrosis factor, PAl-1 - plasminogen activator inhibitor-1. 
did not report more precise data concerning atrial fibrillation (paroxysmal, persistent, chronic AF?). The diagnosis of AF was made on the basis of the surface standard 12 lead electrocardiogram (ECG), and a 24-h Holter monitor examination was not performed, so it was not possible to detect all the AF cases, a fact that may indicate latent bias in this meta-analysis. Third, we observed significant heterogeneity in our meta-analysis and, due to the limited number of studies, a meta-regression model could not be conducted to explore heterogeneity. Fortunately, the heterogeneity disappeared in the subgroup analysis of diabetes.

\section{Conclusions}

Our meta-analysis suggested that ultrasound-diagnosed NAFLD patients have a significantly higher risk for $\mathrm{AF}$ and the increased risk was present predominantly in patients with type 2 diabetes after adjustment for numerous important clinical risk factors for AF. Further large prospective studies are needed to clarify which subgroups of subjects with NAFLD are at higher risk for $\mathrm{AF}$ and should focus on understanding the exact mechanisms for AF risk, on developing reliable tools for risk stratification, and ultimately on devising strategies to prevent AF event in subjects with NAFLD. During the interim, it is even more important that gastroenterologists who start seeing NAFLD especially complicated with diabetes at an earlier stage initiate risk mitigation treatments than cardiologists should be aware of AF.

\section{Conflict of interest}

The authors declare no conflict of interest.

\section{References}

1. Anstee QM, McPherson S, Day CP. How big a problem is non-alcoholic fatty liver disease? BMJ 2011; 343: d3897.

2. Vernon G, Baranova A, Younossi ZM. Systematic review: the epidemiology and natural history of non-alcoholic fatty liver disease and non-alcoholic steatohepatitis in adults. Alimentary Pharmacol Ther 2011; 34: 274-85.

3. Younossi ZM, Stepanova M, Afendy $M$, et al. Changes in the prevalence of the most common causes of chronic liver diseases in the United States from 1988 to 2008. Clin Gastroenterol Hepatol 2011; 9: 524-30.

4. Allen AM, Larson JJ, Therneau TM, et al. The epidemiology of nonalcoholic fatty liver disease (NAFLD) in a US community. Hepatology 2016; 63: 530A-1A.

5. Ratziu V, Bellentani S, Cortez-Pinto $\mathrm{H}$, et al. A position statement on NAFLD/NASH based on the EASL 2009 special conference. J Hepatol 2010; 53: 372-84.

6. Targher G, Day CP, Bonora E. Risk of cardiovascular disease in patients with nonalcoholic fatty liver disease. N Engl J Med 2010; 363: 1341-50.

7. Ballestri S, Lonardo A, Bonapace S, et al. Risk of cardiovascular, cardiac and arrhythmic complications in patients with non-alcoholic fatty liver disease. World J Gastroenterol 2014; 20: 1724-45.
8. Bhatia LS, Curzen NP, Calder PC, et al. Non-alcoholic fatty liver disease: a new and important cardiovascular risk factor? Eur Heart J 2012; 33: 1190-200.

9. Mantovani A, Ballestri S, Lonardo A, et al. Cardiovascular disease and myocardial abnormalities in nonalcoholic fatty liver disease. Dig Dis Sci 2016; 61: 1246-67.

10. Chen YH, Chen KY, Lin HC. Non-alcoholic cirrhosis and the risk of stroke: a 5-year follow-up study. Liver Int 2011; 31: 354-60.

11. Fallo F, Dalla Pozza A, Sonino N, et al. Non-alcoholic fatty liver disease is associated with left ventricular diastolic dysfunction in essential hypertension. Nutrition Metabolism Cardiovasc Dis 2009; 19: 646-53.

12. Goland S, Shimoni S, Zornitzki T, et al. Cardiac abnormalities as a new manifestation of nonalcoholic fatty liver disease: echocardiographic and tissue Doppler imaging assessment. J Clin Gastroenterol 2006; 40: 949-55.

13. Haddad TM, Hamdeh S, Kanmanthareddy A, et al. Nonalcoholic fatty liver disease and the risk of clinical cardiovascular events: a systematic review and meta-analysis. Diabetes Metab Syndr 2016; doi: 10.1016/j/dsx.2016.12.033.

14. Haddad TM, Hamdeh S, Narayanan MA, et al. Nonalcoholic fatty liver disease and the risk of clinical cardiovascular events: a meta-analysis. Circulation 2015; 132.

15. Liu H, Lu HY. Nonalcoholic fatty liver disease and cardiovascular disease. World J Gastroenterol 2014; 20: 8407-15.

16. The Newcastle-Ottawa Scale (NOS) for assessing the quality of nonrandomised studies in meta-analyses. Available at: http:// www.medicine.mcgill.ca/rtamblyn/Readings/The\%20Newcastle\%20-\%20Scale\%20for\%20assessing\%20the\%20quality\%20 of\%20nonrandomised\%20studies\%20in\%20meta-analyses.pdf

17. Dauriz M, Mantovani A, Pichiri I, et al. Non-alcoholic fatty liver disease is associated with an increased prevalence of atrial fibrillation in patients with type 2 diabetes. Diabetes 2013; 62: A371.

18. Karajamaki AJ, Patsi OP, Savolainen M, et al. Non-alcoholic fatty liver disease as a predictor of atrial fibrillation in middle-aged population (OPERA Study). PLoS One 2015; 10: e0142937.

19. Markus MR, Meffert PJ, Baumeister SE, et al. Association between hepatic steatosis and serum liver enzyme levels with atrial fibrillation in the general population: The Study of Health in Pomerania (SHIP). Atherosclerosis 2016; 245: 123-31.

20. Targher G, Valbusa F, Bonapace S, et al. Non-alcoholic fatty liver disease is associated with an increased incidence of atrial fibrillation in patients with type 2 diabetes. PLoS One 2013; 8: e57183.

21. You SC, Yang PS, Kim TH, et al. Non-alcoholic fatty liver disease is independently associated with new onset atrial fibrillation: a nationwide cohort study in korea. J Am Coll Cardiol 2016; 67: 854.

22. Hernaez R, Lazo M, Bonekamp S, et al. Diagnostic accuracy and reliability of ultrasonography for the detection of fatty liver: a meta-analysis. Hepatology 2011; 54: 1082-90.

23. Sookoian S, Pirola CJ. Non-alcoholic fatty liver disease is strongly associated with carotid atherosclerosis: a systematic review. J Hepatol 2008; 49: 600-7.

24. Bonnet F, Gastaldelli A, Pihan-Le Bars F, et al. Gamma-glutamyltransferase, fatty liver index and hepatic insulin resistance are associated with incident hypertension in two longitudinal studies. J Hypertens 2017; 35: 493-500.

25. Liu J, Au Yeung SL, Lin SL, et al. Liver enzymes and risk of ischemic heart disease and type 2 diabetes mellitus: a Mendelian randomization study. Sci Rep 2016; 6: 38813. 
26. Ndrepepa G, Kastrati A. Gamma-glutamyl transferase and cardiovascular disease. Ann Translat Med 2016; 4: 481.

27. Ndrepepa G, Xhepa E, Colleran R, et al. Gamma-glutamyl transferase and atrial fibrillation in patients with coronary artery disease. Clin Chim Acta 2017; 465: 17-21.

28. Minhas AM, Usman MS, Khan MS, et al. Link between non-alcoholic fatty liver disease and atrial fibrillation: a systematic review and meta-analysis. Cureus 2017; 9: e1142.

29. Schnabel RB, Sullivan LM, Levy D, et al. Development of a risk score for atrial fibrillation (Framingham Heart Study): a community-based cohort study. Lancet 2009; 373: 739-45.

30. Sinner MF, Wang N, Fox CS, et al. Relation of circulating liver transaminase concentrations to risk of new-onset atrial fibrillation. Am J Cardiol 2013; 111: 219-24.

31. Rajappa M, Goswami B, Balasubramanian A, et al. Interplay between inflammation and hemostasis in patients with coronary artery disease. Indian J Clin Biochem 2015; 30: 281-5.

32. Schrör K, Huber K. Platelets, inflammation and anti-inflammatory drugs in ACS and CAD. Thromb Haemostasis 2015; 114: 446-8.

33. Ndumele CE, Nasir K, Conceicao RD, et al. Hepatic steatosis, obesity, and the metabolic syndrome are independently and additively associated with increased systemic inflammation. Arterioscler Thromb Vasc Biol 2011; 31: 1927-32.

34. Targher G, Bertolini L, Rodella S, et al. NASH predicts plasma inflammatory biomarkers independently of visceral fat in men. Obesity 2008; 16: 1394-9.

35. Byrne CD, Targher G. Ectopic fat, insulin resistance, and nonalcoholic fatty liver disease: implications for cardiovascular disease. Arterioscler Thromb Vasc Biol 2014; 34: 1155-61.

36. lacobellis G, Barbarini G, Letizia C, et al. Epicardial fat thickness and nonalcoholic fatty liver disease in obese subjects. Obesity 2014; 22: 332-6.

37. Kato K, Takamura T, Takeshita Y, et al. Ectopic fat accumulation and distant organ-specific insulin resistance in Japanese people with nonalcoholic fatty liver disease. PLoS One 2014; 9: e92170.

38. Mehta SR, Thomas EL, Bell JD, et al. Non-invasive means of measuring hepatic fat content. World J Gastroenterol 2008; 14 3476-83.

39. Farrell GC, Larter CZ. Nonalcoholic fatty liver disease: from steatosis to cirrhosis. Hepatology 2006; 43 (2 Suppl. 1): S99-112. 